\title{
Regulatory testing for safety: The mathematics of broad-based results
}

T. P. Hutchinson ${ }^{1} \quad$ R. W. G. Anderson ${ }^{2} \quad$ D. J. Searson ${ }^{3}$

(Received 10 December 2015; revised 8 August 2016)

\begin{abstract}
Testing of the safety of manufactured products is typically conducted under a specified set of conditions. For example, when projecting an instrumented headform at the front of a car to assess the pedestrian safety of that model of car, the speed of the headform is specified. But surely, if they were asked, the public and policymakers would say that the result at one speed is a rather artificial measure, and they instead wish to know the average level of safety across real-world impact scenarios. One possible solution is to directly test across the range of conditions and combinations of conditions. However, manufacturers typically want to economise by conducting fewer tests. This article considers how to determine a product's safety for a range of conditions, while also being economical with the testing. What is proposed has
\end{abstract}

DOI:10.21914/anziamj.v57i0.10332 gives this article, (c) Austral. Mathematical Soc. 2016. Published September 18, 2016, as part of the Proceedings of the 12th Biennial Engineering Mathematics and Applications Conference. ISSN 1445-8810. (Print two pages per sheet of paper.) Copies of this article must not be made otherwise available on the internet; instead link directly to the DOI for this article. Record comments on this article via http://journal austms.org.au/ojs/index.php/ANZIAMJ/comment/add/10332/0 
three steps. The first is to generalise the quantity observed in test conditions to what would be observed under different conditions. This is likely to involve a theory and a formula. For example, in a headform impact test the quantity observed might be HIC (the Head Injury Criterion), the condition that varies might be impact speed, and a formula might be available for the dependence of HIC on speed. The second is to convert the test quantity to something that is meaningfully averaged. This might be the dollar cost associated with a particular level of HIC, or perhaps the probability of death. The third is to obtain the average, by integration over the condition that varies from crash to crash (such as impact speed). In principle, this procedure is quite general and applicable to many other forms of testing. Good information is required for the three steps, but this is inherent in aiming for a broad-based result, rather than due to the method.

\section{Contents}

1 Introduction

2 Proposed method

3 Equation for average cost

3.1 Notation . . . . . . . . . . . . . . . . . C197

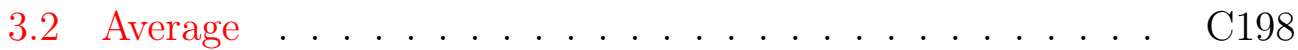

3.3 Theory for the dependence of the test result on the inputs C199

3.4 Example of calculation . . . . . . . . . . . . C200

3.5 Range of validity of $h(x) \ldots \ldots \ldots$ C201

4 Two analogous calculations

5 Discussion 
5.2 Application to integrated assessment of primary and secondary safety

C203

5.3 Frailty . . . . . . . . . . . . . . . C204

5.4 Concluding comments . . . . . . . . . . C204

\section{References}

C205

\section{Introduction}

A lot of testing is carried out to ensure consumer products are safe. For example, as part of testing of new cars by consumer organisations, an approximate sphere with an accelerometer inside it is projected at the front of a car. The acceleration pulse is recorded. This gives a guide to the likely injury severity of a pedestrian struck by the car and the quantity calculated is termed the Head Injury Criterion (HIC) [7].

Speed and other conditions of the impact are specified; here we explore the contrast between these specifications and the reality that pedestrian impacts occur over a wide range of speeds and other conditions. This contrast occurs in many other types of test. Here we sometimes write in terms of impact testing, and sometimes in general terms.

We identify the following issues.

- The need to do lots more tests in order to obtain the basic data on how HIC varies with speed - or, alternatively, a theory about how HIC depends on speed is required.

- While it is hoped that HIC reflects or indicates likely injury, it is not itself a measure of injury.

- It is unlikely that it is sensible to average HIC. One person with $\mathrm{HIC}=900$ and one with $\mathrm{HIC}=1100$ is about as bad an outcome as two people with $\mathrm{HIC}=1000$, but this simple averaging is arguably 
inappropriate for one person with $\mathrm{HIC}=200$ and one person with $\mathrm{HIC}=1800$.

- Information about frequencies of impacts at different speeds is needed. We use the term "regulatory testing" for convenience; a lot of valuable testing does not have the full force of laws and regulations behind it, but may be done (for example) for consumer information purposes. This is the case for the pedestrian headform testing that we refer to here. One possible method-perhaps the best method - of determining the level of safety in a range of scenarios is to test across the range of scenarios and combinations of scenarios. In some cases, this is done: many different locations on a car exterior are tested. Nothing in this article should be taken as critical of that straightforward approach. But it may be possible to use theory to economise on the number of tests.

\section{Proposed method}

What is of chief interest in car impact tests is the injury. What is measured in an impact test is the acceleration pulse, and this serves as a proxy for the injury. A contrast like this between what is of central interest and some measurable physical quantity is a feature of many other types of tests. An acceleration pulse in an impact test is usually summarised by a single number - a calculation is carried out that results in the HIC (in some other test protocols, the maximum acceleration is used instead). As already noted, tests could be conducted at a number of different speeds. Alternatively, there may be some theory available concerning the dependence of HIC on speed.

Suppose that results are obtained, whether directly by testing or by some other method, at lots of different speeds. It is often impossible to understand so many results, and they need to be summarised. That is, an average needs to be calculated. Thus the results need to be numbers (and not words such as 'Good' or 'Unsatisfactory'). 
It is likely that decisions are taken based on a set of test results. For example, whether the tested car has passed or not, or whether this car is better than that car. Therefore the number associated with a single test needs to be such that when several of these numbers are obtained, a decision can be made based on an average. That is, final results from tests need to be 'utilities' or 'values' or 'costs'.

The proposed calculations are as follows [6].

1. From the acceleration pulse obtained at one speed (or under one set of conditions), the HIC is calculated.

2. Either the HIC measurement is repeated for several speeds, or the results for various speeds are found using theory.

3. Each HIC is converted to the corresponding cost.

4. From the costs at several speeds (or in several sets of conditions) together with the probabilities of these speeds occurring in the real world, the average cost is calculated.

For decades there has been some dissatisfaction with the limitations of a single set of car test conditions [6]. The relation between a test result and real-world performance is of broad interest, but we know of little that is analogous to the measure-generalise-cost-average sequence just outlined.

\section{Equation for average cost}

\section{$3.1 \quad$ Notation}

Notation is given below.

- $x$ : speed of impact of the car with the pedestrian (assumed to be the same as that with which the head hits the car); more generally, this is 
any quantity that is specified for a test but varies in the real world.

- $h(x)$ : HIC, the Head Injury Criterion; more generally, this is the outcome of the test.

- $p(h)$ : cost or utility associated with the test outcome $h$ (the clinical nature of injury and the outcome vary randomly between different people, and in that sense this is an average); this is the quantity on which decisions are based.

- $f(x)$ : probability density function of $x$.

In the case of pedestrian injury, $p$ may be a true average dollar cost, including amounts for the 'value of life' and for pain and suffering, but at the present state of knowledge it may be something simpler, such as the probability of death at a specified value of $h$.

Functions $p(h)$ and $f(x)$ are difficult to determine empirically, but Hutchinson et al. [6] made and used estimates.

\subsection{Average}

The test takes place at some particular speed $v$ (e.g., $40 \mathrm{~km} / \mathrm{h}$ ), and HIC $\mathrm{h}$ is observed. There is some function $h(x)$ describing HIC at other speeds $x$. The average cost is

$$
\operatorname{Av}(p)=\int p(h(x)) f(x) d x .
$$

Locations on the car differ in how safe or unsafe they are, and the above equation refers to any particular location on the car. However, it may be desired to average over the whole car, and also over pedestrian head mass and stature. Thus $x$ may not be a single quantity but instead a vector of quantities such as speed of impact, effective mass of the pedestrian's head, stature of the pedestrian, and so on. 
The following puts equation (1) into words. Consider all conditions (in this example, all speeds of impact). Assume that it is known how frequently each occurs. On the basis of a test result in one condition and a theory, work out the test result in all conditions. Then convert each of these to a cost (that is, a number representing how bad it is). Use the frequencies of the conditions to average these costs.

\subsection{Theory for the dependence of the test result on the inputs}

Equation (1) economises on the number of tests by substituting a theoretical function $h(x)$, based on one test result, in place of empirical observations.

When referring to impacts, as this example does, the dependent variables of most interest are:

- HIC, maximum acceleration, maximum force - these reflect the severity of injury that would be sustained;

- maximum displacement (distance of deformation);

- duration of the acceleration pulse.

There is surprisingly little theory available describing the relationship between HIC and impact speed. A theory about the dependence of HIC on impact speed probably needs to originate in a theory about the force between two bodies in impact. For example, instantaneous force might be assumed to be proportional to instantaneous displacement of the impacted body, as for the linear spring. Maximum acceleration of the impacted body is then proportional to impact speed. If the linear spring is thought to be an inapropriate assumption, then force proportional to some power of displacement might be assumed instead. Let $w(t)$ be displacement, and assume force is proportional to $w^{c}$. Using the principle of conservation of energy, it is then elementary to show that maximum acceleration, maximum force, and maximum displacement are all 
proportional to some power of impact speed. That leaves open the question of whether a similar result holds for HIC.

More generally, and using dashes for time derivatives, the following differential equation relates acceleration $w^{\prime \prime}$ (and force $m w^{\prime \prime}$ ) to instantaneous deformation (distance) $w$ and instantaneous velocity $w^{\prime}[3,2]$ :

$$
m w^{\prime \prime}-k w^{c}\left[1+(b / v) w^{\prime}\right]=0 .
$$

If differential equation (2) is correct, then several dependent variables (including HIC, maximum acceleration, maximum force, maximum displacement, and duration of the acceleration pulse) are proportional to power functions of $m / k$ and of $v[4]$. For example,

$$
\mathrm{HIC} \propto(\mathrm{m} / \mathrm{k})^{-1.5 /(\mathrm{c}+1)} v^{(4 c+1) /(\mathrm{c}+1)} .
$$

If $\mathrm{c}$ is approximately one, then the exponent of $v$ is approximately 2.5.

\subsection{Example of calculation}

Table 1 is an example of the mechanics of calculation. Instead of a continuous probability density function for $f(x)$, there is a discrete distribution over three categories. We suppose that three values of $x$ (in column 2) occur with probabilities (column 1) 0.5, 0.3, and 0.2, respectively. Column 3 of the table shows the single value of $h$ that was observed experimentally, at $x=40$. Column 4 shows the values of $h$ determined theoretically. Here, we choose $x$ to be $v$ and $h$ to be HIC as given in equation (3), and assume that $c=1$. Column 5 shows the costs (disutilities) associated with the respective values of $h$ (indicative round numbers here, but determined empirically in a real calculation). Finally, column 6 shows the products pf, the total of which is $\operatorname{Av}(p)=135$. 
Table 1: Example of use of equation (1). For further explanation, see Section 3.4.

\begin{tabular}{rrrrrr}
1 & 2 & 3 & 4 & 5 & 6 \\
$\mathrm{f}$ & $x$ & $\mathrm{~h}$ & $\mathrm{~h}$ & $\mathrm{p}$ & $\mathrm{pf}$ \\
\hline 0.5 & 20 & & 88 & 10 & 5 \\
0.3 & 40 & 500 & & 100 & 30 \\
0.2 & 60 & & 1378 & 500 & 100
\end{tabular}

\subsection{Range of validity of $h(x)$}

With blunt impact, an important limitation on the validity of $h(x)$ is the potential occurrence of 'bottoming out'. This term refers to the car bonnet deforming so much that very stiff structures underneath it are contacted, resulting in a significant increase of the deceleration of the pedestrian's head and of the probability of very severe injury.

\section{Two analogous calculations}

Equation (1) might be relevant to many fields of testing, if there is appropriate interpretation of the functions $h(\cdot), p(\cdot)$, and $f(\cdot)$. Two similar sets of calculations are summarised here.

The first set of calculations are about inferring from a small number of tests of a specified missile (into a tissue simulant) what the average effect would be on the human body [8]. Hutchinson's [5] interpretation of Kokinakis and Sperrazza's [8] data was that $h(x)$ is the wound received (the name of the injury, or a description) when someone is struck at location $x$ (the name of the part of the body), $p(h)$ is the level of incapacitation (a percentage), $f(x)$ is the probability of $x$ being the part of body that is hit, and equation (1) gives the average level of incapacitation. There is continuing interest in this problem. For example, VanAmburg [9] estimated the likely level of injury 
from a fragment at different impact points on the limbs or face; the calculation was then taken a step further, to give a probability over a random point of impact of a specified level of injury. In equation (1) the conversion of $h$ to $p$ only has one stage; however, in the vulnerability/lethality context, "target damaged components" lead to "target measures-of-capability" which in turn lead to "target measures-of-effectiveness (utility)" [1].

The second set of calculations were discussed by Webby et al. [10 ${ }^{1}$. A sequence of calculations concerning flooding of the Mekong river in Cambodia [10] has some similarities with equation (1). Flooding has benefits as there is a positive dependence of fish catch (thousands of tonnes) on flood volume (hundreds of cubic kilometres). Flood volume might be taken as the random variable $x$, fish catch as the dependent variable $h(x)$, and historical data give an estimate of the probability density $f(x)$. Economic consequences for the fishery are $p(h)$. Webby et al. [10] assumed $p$ proportional to $h$. However, a non-linear dependence can be imagined because of decreasing unit price when supply is plentiful. Alternatively, $p$ might represent the cost to an international aid agency, being zero in normal times and large when the flood is greatly below normal. Webby et al. [10] considered aid expenditure that is dependent on $x$ rather than $h$.

\section{Discussion}

\subsection{Possible interaction of design and speed}

One of the purposes of testing is to permit comparison of one model of car with another. Even if a test procedure were inaccurate as regards absolute level of risk, it might nevertheless be very useful if it provided a fair comparison. Thus the question arises, if one model of car performs better than another in the test, then does it also perform better in similar tests conducted at lower

\footnotetext{
${ }^{1}$ We thank a referee for drawing our attention to this.
} 
and higher speeds? Lack of consistency in this respect and the effects of this inconsistency is referred to as interaction between car design and speed.

Such interaction is possible. Suppose that model A gives rise to a lower HIC than model B at the standard test speed, but the bonnet of model A is close to bottoming out, whereas there is spare space available for further deformation under the bonnet of model B. Then at a higher test speed, model A is likely to be much worse than at the standard speed, whereas model B will be only a little worse, and model A may now give rise to the higher HIC.

In principle, equation (1) permits a relatively poor low-speed performance that worsens only slightly with increasing speed, as well as the contrasting case which has a relatively good low-speed performance that unfortunately worsens sharply with increasing speed (the practical difficulty is that the function $h(x)$ is very poorly understood in the case of bottoming out).

\subsection{Application to integrated assessment of primary and secondary safety}

Probabilities $f(x)$ specify how common are bad conditions, and the function $h(x)$ specifies the effect of bad conditions on the object under test. Both $f$ and $h$ may change. Improvements to braking systems or tyres, and new technologies such as autonomous braking, may substantially reduce impact speeds: a change in the distribution of speeds, $f(x)$. Change to the design of the vehicle bonnet and to the stiff structures underneath are what affect the impact test result $h(x)$. Equation (1) depends on both $f(x)$ and $h(x)$, and thus permits the integrated assessment of both primary and secondary safety features. There is no suggestion that secondary safety requirements should be relaxed for cars with good primary safety; rather, it is envisaged that when improved primary safety becomes common in new cars, cars that lack those features should be subject to tightened secondary safety requirements.

From Equation (3), the ratio of benefits from a one percent reduction in 
impact speed to those from a one percent reduction in the HIC observed in a test is $(4 c+1) /(c+1)$. This ratio is between one and four, and is 2.5 if $c=1$.

Consider the example in Table 1 again. but now suppose that when $x$ is $40, h$ is 550, which is a little worse. Corresponding to $x=(20,40,60)$, the vector $h$ is predicted to be $(97,550,1516)$. Assume the costs $p$ are $(12,120,600)$. Now suppose that the probabilities $f$ are $(0.7,0.2,0.1)$. The sum of the three values of the product pf is $8.4+24+60=92.4$. This sum is lower than the total of 135 in Table 1: the change in the distribution of $x$ has (in this example) more than compensated for the increase in $h$.

\subsection{Frailty}

The frailty of the person struck is important in determining the outcome, but is not represented in equation (1). Frailty is typically seen as outside the scope of the testing context, as a process of averaging over people occurs in the construction of the $p(h)$ function. If, on the other hand, it were thought that the distribution of impact speeds $f(x)$ were different for people of different frailties (of different ages, for example), then it would be necessary or desirable to represent frailty explicitly in the equation.

\subsection{Concluding comments}

Equation (1) itself is simple once the principle is appreciated. But to some extent it is an aspiration rather than a reality, as its components $h, p$, and $f$ are difficult to determine. In the headform impact context, it is not impossible to get some information on these functions, and this might be easier in some other fields of application. Equation (1) at least draws attention to the many cases occurring at low speeds of impact and the very high severity of the cases occurring at high speeds of impact. 
In car safety testing, where might mathematics be most useful? In the pedestrian injury context, $p(\cdot)$ and $f(\cdot)$ are empirical, but $h(\cdot)$ is theoretical (unless more testing is done). It would be useful to have more differential equations that are reasonably plausible and permit derivation of relationships for the dependent variables of interest. That applies to both ordinary impacts that are designed for, and impacts in which there is bottoming out. That is, alternatives to equation (2) and derived proportionality relationships, such as equation (3), would be welcome.

Acknowledgements The Centre for Automotive Safety Research (CASR) receives core funding from both the South Australian Department for Planning, Transport and Infrastructure and the South Australian Motor Accident Commission. CASR performs pedestrian impact tests for the Australasian New Car Assessment Program (ANCAP). The views expressed are those of the authors and do not necessarily represent those of the University of Adelaide, ANCAP, or CASR's other funding organisations.

\section{References}

[1] P. H. Deitz. A V/L taxonomy for analyzing ballistic live-fire events. Proceedings of the 15th International Symposium on Military Operational Research, 1998. http://ismor.cds.cranfield.ac.uk/15th-symposium-1998 C202

[2] R. G. Herbert and D. C. McWhannell. Shape and frequency composition of pulses from an impact pair. J. Eng. Ind. 99:513-518, 1977. doi:10.1115/1.3439270 C200

[3] K. H. Hunt and F. R. E. Crossley. Coefficient of restitution interpreted as damping in vibroimpact. J. Appl. Mech. 42:440-445, 1975. doi:10.1115/1.3423596 C200 
[4] T. P. Hutchinson. Dependence of the Head Injury Criterion and maximum acceleration on headform mass and initial velocity in tests simulating pedestrian impacts with vehicles. J. Biomech. Eng. 135:114508, 2013. doi:10.1115/1.4025331 C200

[5] T. P. Hutchinson. Experimental injury: Inference from proxy observations in a test to the real-world average. J. Battlefield Tech. 18(1):1-6, 2015. http://search.informit.com.au/documentSummary; $\mathrm{dn}=958266262006914$; res=IELENG C201

[6] T. P. Hutchinson, R. W. G. Anderson and D. J. Searson. Pedestrian headform testing: Inferring performance at impact speeds and for headform masses not tested, and estimating average performance in a range of real-world conditions. Traffic Inj. Prev. 13:402-411, 2012. doi:10.1080/15389588.2012.660252 C197, C198

[7] T. P. Hutchinson, D. J. Searson, R. W. G. Anderson, J. K. Dutschke, G. Ponte and A. L. van den Berg. Protection of the unhelmeted head against blunt impact: The pedestrian and the car bonnet. Proceedings of the Australasian Road Safety Research, Policing and Education Conference, 2011.

http://acrs.org.au/files/arsrpe/Protection $\% 20$ of $\% 20$ the $\%$ 20unhelmeted\%20head\%20against\%20blunt\%20impact.pdf C195

[8] W. Kokinakis and J. Sperrazza. Criteria for incapacitating soldiers with fragments and flechettes. Technical Report 1269, Ballistic Research Laboratories, Aberdeen Proving Ground, MD, 1965. http://www.dtic.mil/dtic/tr/fulltext/u2/359774.pdf C201

[9] R. VanAmburg. An approach to analyze personnel injury of reflective spall from small-arms protective body armor. Technical Report ARL-TR-5595, U.S. Army Research Laboratory, Aberdeen Proving Ground, MD, 2011. http://www.dtic.mil/dtic/tr/fulltext/u2/a550618.pdf C201 
[10] R. B. Webby, P. T. Adamson, J. Boland, P. G. Howlett, A. V. Metcalfe and J. Piantadosi. The Mekong - applications of value at risk (VaR) and conditional value at risk (CVaR) simulation to the benefits, costs and consequences of water resources development in a large river basin. Ecological Modelling, 201:89-96, 2007. doi:10.1016/j.ecolmodel.2006.07.033 C202

\section{Author addresses}

1. T. P. Hutchinson, Centre for Automotive Safety Research, University of Adelaide, South Australia 5005, Australia. mailto:paul@casr.adelaide.edu.au orcid:0000-0002-4429-0885

2. R. W. G. Anderson, Centre for Automotive Safety Research, University of Adelaide, South Australia 5005, Australia.

3. D. J. Searson, Centre for Automotive Safety Research, University of Adelaide, South Australia 5005, Australia. 